\title{
Oltre la ricerca del Graal, il divenire del mito
}

\author{
Alberto Castaldini
}

\author{
Center for Hebrew Studies, University of Bucharest \\ acastaldini@libero.it \\ http://orcid.org/OOOO-OOO3-2068-5492 \\ Rebut II/o6/20I4; acceptat 27/o7/20I4 \\ DOI Io.7203/MCLM.I.3806
}

\section{Beyond the Search of the Grail: the Becoming of the Mith}

Abstract

A mythologist's study of the Graal cultural tradition. Using Francesco Zambon's recent Metamorfosi del Graal as his starting point, the author undertakes an analysis on the importance of the Graal in Western mystical, philosophical and literary traditions throughout time, from the impact of the medieval Graal stories on the matière de Bretagne and beyond (Troyes, Boron), to the appeal of this myth in modern times, including its occurrence on Richard Wagner, Martin Heidegger, Simone Weil, Italo Calvino and Umberto Eco.

\section{KeYwords}

Myths; Grail; matière de Bretagne; Arthurian myths; mythologies; gnoseology; philosophical traditions; imaginary; literary sources; religions; Perceval; Galahad; Joseph of Arimathea; Chrétien de Troyes; Robert de Boron; Martin Heidegger; Wolfram von Eschenbach; Richard Wagner; Simone Weil; Italo Calvino; Umberto Eco; Francesco Zambon.

\section{$\Rightarrow * \approx$}

Abstract

Uno studio mitologico sulla tradizione culturale del Graal. Utilizzando come punto di avvio il recente saggio di Francesco Zambon Metamorfosi del Graal, l'autore intraprende un'analisi sull'importanza del Graal nella tradizione mistica, filosofica e letteraria dell'Occidente. Lo fa in una prospettiva diacronica: dall'impatto delle storie medievali del Graal sul Ciclo bretone e oltre (Troyes, Boron), al fascino esercitato da questo mito nei tempi moderni, come la sua presenza nelle opere di Richard Wagner, Martin Heidegger, Simone Weil, Italo Calvino e Umberto Eco.

\section{Parole chiave}

Miti; Graal; matière de Bretagne; miti arturiani; mitologie; gnoseologia; tradizioni filosofiche; immaginario; fonti letterarie; religioni; Perceval; Galaad; Giuseppe di Arimatea; Chrétien de Troyes; Robert de Boron; Martin Heidegger; Wolfram von Eschenbach; Richard Wagner; Simone Weil; Italo Calvino; Umberto Eco; Francesco Zambon.

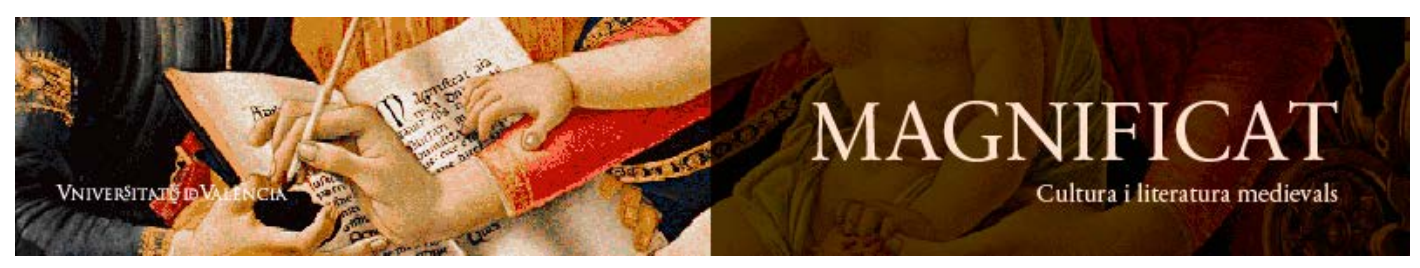

Magnificat Cultura i Literatura Medievals I, 20I4, 37-50. http://ojs.uv.es/index.php/MCLM

ISSN 2386-8295 
Alberto Castaldini, 20I4, "Oltre la ricerca del Graal, il divenire del mito", Magnificat Cultura i Literatura Medievals $\mathrm{I}, 37^{-5} \mathrm{O}$. (๕) Ev

\title{
ə*⿻丷木
}

\begin{abstract}
Al Graal, mito par excellence della letteratura cavalleresca e cortese, è dedicato il recente volume di Francesco Zambon, dal titolo fortemente evocativo: Metamorfosi del Graal (Zambon 2OI2). Il saggio, preziosa sintesi di vent'anni di ricerca filologica e letteraria, ha ispirato questo contributo. La rigorosa analisi, che in diciotto capitoli attraversa un millennio di letteratura europea, presenta inevitabili suggestioni esoteriche dalle quali crediamo l'autore sia stato accarezzato, ma a cui necessariamente si è sottratto pur senza rinunciarvi nell'intimo. Il libro possiede peraltro il pregio di un titolo ri-velatore, in apparenza essoterico, che al lettore potrebbe apparire come risolutivo l'antica quaestio su che cosa realmente sia e dove effettivamente si trovi il Graal. Esso ci sembra infatti la chiave (la cléf, per riandare a Eliphas Lévi), o il Leitmotiv (per dirla sulle note del "graalico" Richard Wagner) che riannoda alla fine la ricca trama testuale e intepretativa dell'intero lavoro, senza però negarne ulteriori e possibili sviluppi.
\end{abstract}

Il segreto del Graal sta forse nella sua metamorfosi: una metamorfosi/divenienza continua, pressoché incontrollabile. Di conseguenza ogni percorso iniziatico, umanistico e religioso, intrapreso per raggiungerlo, non può avere compimento perché sarebbe impossibile individuarlo e riconoscerlo. Sin dall'inizio il suo pieno possesso risulta dunque vano.

Ciò però non significa che la sua ricerca, anche quella letteraria, sia inutile: se il Graal appartiene al novero delle "cose nascoste sin dalla fondazione del mondo" (Matteo I3,35), non è concesso all'uomo negarne l'esistenza, spegnerne il desiderio, rinunciarvi. Se il mito racchiude una dimensione ontologica inesauribile, è di qualsiasi fede il contenuto ineffabile, eppure così necessario nella tragica condizione terrena, dove il lógos, per non inaridirsi, si alimenta della fantasia. ${ }^{.}$Per questo la ragione non deve temere né il mito e nemmeno il Nulla (simile all' En-Sof primigenio dei cabalisti), condizione per la pensabilità dell'incondizionato, dell'assoluto, nella sua libertà creatrice. Come ha scritto il filosofo Sergio Givone, è il Nulla "che restituisce l'essere a se stesso", a ricondurlo ad una condizione estatica e perciò alla più autentica libertà (Givone 2006 , 38).

Il Graal appare essenzialmente metamorfico proprio nella sua infinita libertà ontogena, che è prima di tutto sorgente della vita, tragica e inesauribile, Dasein illimitato, antitetico per esempio al Niente barthiano, il "das Nichtige", cioè il "Niente reale, il Nemico, colui che dice-no al mondo" (Cacciari 2004, 358) e ancor prima alla vita.

I. Platone del resto usava mythos e lógos con mutevoli accezioni, come nel Fedone (Jesi I989, I7). 
È quella del Graal una “cerca” (queste) che "affonda le sue radici nei secoli” (Zambon 20I2, IO). Un tema originario costitutivo, un archetipo, poi legatosi al mondo cavalleresco, che innerva un epos millenario: da Chrétien de Troyes a Robert de Boron, a Wolfram von Eschenbach, per citare gli aedi principali dell'impresa. Questo perché l'inesauribile vocazione dei cavalieri Parsifal e Galaad maturò già in sella ai cavalli protostorici delle pianure eurasiatiche emerse dalle nebbie paleolitiche, si prefigurò nelle visioni sciamaniche come nei riti di passaggio dalla Urheimat indoeuropea a quello che oggi chiamiamo l'Occidente, con le sue "strutture" sociali, politiche e religiose.

L'ideologia tripartita del "sistema” indoeuropeo fissata da Georges Dumézil (sacerdoti, guerrieri, agricoltori) ${ }^{2}$ prefigura quella dell'ordo medievale cristiano: rigido per nascita ma scardinabile da una scelta-ricerca-vocazione esistenziale, perfezionabile da una conquista. Il coraggio della "cerca" trasformava in cavalieri, la vocazione della fede in preti e monaci.

Quello del viaggio e dell'impresa di pochi valorosi per la salvezza di tutti, diventò così un passo obbligato nella trasformazione della società nomade in regno stanziale, della tribù in comunità, il gesto fondativo di ogni codice comportamentale, individuale e collettivo, suggellato dalla perfectio evangelica (la rivoluzione di Gesù, nuovo Adamo e nuovo Mosè) che fu alla base della Christianitas e che trasformò la militia saeculi in militia Dei.

Di questa conversio culturale oltre che religiosa Bernardo di Chiaravalle fu nel secolo XII l'iniziatore e il garante. E ne indicò chiaramente la mèta: Gerusalemme, con i resti del suo antico Tempio, "domus Dec", distrutto un millennio prima, poi interiorizzato dal giudaismo diasporico, luogo di preghiera per tutte le genti (Isaia, 2,2), prefigurazione del trono divino (divenuto trinitario) nei cieli.

Un luogo santo andava soprattutto visitato in quella città: il Santo Sepolcro, nuovo sancta sanctorum, memoria del Qodesh ha-Qodashim, "reliquia per eccellenza", "che tiene fra tutte il principatum" (Zambon 2OI2, I3), rinnovato tempio della presenza-assenza del Dio fattosi uomo, al quale potevano avere accesso le schiere di tutti i coraggiosi, cavalieri e pellegrini, che il lungo e pericoloso viaggio, e la battaglia non solo fisica, avevano trasformato nei nuovi sacerdoti e leviti chiamati a custodire e a difendere la "casa di Dio" anche con le armi, persino morendo, condividendo in tal modo quella radicale kenosi del Divino che è la morte nella carne, l'attraversamento delle tenebre.

Ma il Santo Sepolcro non era come il Tempio sede della temuta "presenza" della regalità divina, bensì scandalosa e arrendevole testimonianza del suo abbassamento; non l'ultimo recesso dell'Arca dell'Alleanza, ma l'estrema dimora di un crocifisso prima della grande svolta operata nel tempo: la "Kehre" della Redenzione, attraverso quella morte cui è strettamente associata ogni nascita. Scrive san Paolo nella Lettera ai Romani $(6,4)$ : "Per mezzo del battesimo siamo dunque stati sepolti insieme a lui nella morte". Il Sepolcro apparve dunque alla cristianità il luogo in cui si gioca la speranza e l'attesa di una vita nuova.

È Bernardo nel De laude novae militiae (II29-II36) a dedicare una speciale attenzione al tema della morte di Cristo, "volontaria e immeritata". Sembra invece trascurare la resurrezione: questo perché è la morte del Dio incarnato ad aver portato la salvezza all'umanità, è il luogo dove fu riposto il suo corpo che, anche se vuoto, è "pieno di misteri, di gioiosi misteri”. La morte di Cristo è "il vero scopo, interiore e spirituale, della cavalleria templare, ben al di là dei concreti compiti militari che le

2. Tripartizione che secondo lo storico e linguista francese ha esercitato il proprio influsso anche sull'epos del continente (Dumézil ı968). 
sono assegnati” (Zambon 2OI2, I4).

Il viaggio di conversione nella terra della salvezza aveva come scopo principale l'incontro con la morte: senza di essa sarebbe stata infatti impossibile la teokoinia, la comunione reale con Dio. La celebrazione del sacramento eucaristico nelle pievi e nelle cattedrali, e cioè l'assimilazione del corpo divino nel corpo degli uomini, non bastava: era necessario morire concretamente per e con quel corpo. La salvezza è un'impresa che passa necessariamente dalla morte, che accomuna il creatore e le creature: il teomorfismo di Adamo in Genesi si converte nell'antropomorfismo di Dio nei Vangeli ("Egli vi ha riconciliati per mezzo della morte del suo corpo di carne"-Lettera ai Colossesi, I,22).

Cinquant'anni dopo il De laude bernardiano, Chrétien de Troyes scriverà il romanzo fondatore del mito: Le roman de Perceval ou le conte du Graal. Anche in Chrétien non è la resurrezione, superamento della morte e di ogni caducità corporea ad essere celebrata, ma la morte stessa. Il Graal per Chrétien non è una reliquia ma una "sainte chose" (materialmente un piatto) che contiene l'ostia (così descritta per contingenti ragioni liturgico-sacramentali), cioè il corpo del condannato, come una perpetuazione del Sepolcro, e perciò la sua forma si presenta potenzialmente metamorfica.

Il suo mistero è detenuto dal santo eremita presso il quale si recherà Perceval/Parsifal, l'iniziato, prototipo dell'uomo occidentale moderno attraversato dallo smarrimento. Niente a che vedere con l'astuzia e la superbia di Ulisse, piuttosto con la dolorosa ricerca interiore di Virgilio, anche lui in qualche modo inconsapevole profeta di un "graal" (è un’immagine che ci ispira Hermann Broch col suo capolavoro Der Tod des Vergil scritto di fronte alla "waste land" europea del r945). Perceval è il cavaliere chiamato ad un servizio che porterà beneficio a tutti gli uomini: in lui ragione e natura (cioè l'istinto) si fondono in una meraviglia che non è aristotelica ma "eccesso di estasi mistica" di fronte all'Ineffabile, a "ciò che la lingua non può descrivere né cuore immaginare" (Zambon, 2OI2, 2I), come esclamerà Galaad, il “cavaliere celeste”, nella Queste del Saint Graal.

In fondo al Graal, qui descritto come un vaso, Galaad scorge il volto di Dio, contemplato dal cavaliere "facie ad faciem" come avvenne per Mosè in Esodo: "Il Signore parlò a Mosè [faccia a faccia]" (Esodo 33,II)". La "cerca" diviene per l'umanità un "mezzo di avvicinamento e di partecipazione al Divino" (Ponsoye I989, I3), e perciò un nuovo Esodo: viaggio rivelatore e salvifico, "rivoluzionario" perché liberatorio (Walzer I985) e ancora in corso.

Il Graal nel volgere di pochi decenni aveva dunque già compiuto una metamorfosi simbolica e letteraria, lasciando però immutato il desiderio di Dio, l'intellectus amoris che solo lo può conoscere e che in Guglielmo di Saint-Thierry appare la massima forma di conoscenza nell'uomo, "quando si è fatto a somiglianza di Colui che lo ha fatto" (Zambon 2OI2, 242).

Questa perenne e vitale mutazione è il motore della costruzione del discorso epico sul Graal (e perciò della sua ek-sistenza), nonostante ogni dannosa stabilizzazione mitopoietica in nome di credenze o dogmi (come nel caso della transustanziazione), o di interpretazioni positivistiche (come quella di Jessie Weston, I994, 234ss., che sulla scorta di James G. Frazer, scorge nel Graal la sopravvivenza di rituali di rinascita naturale). La finzione romanzesca è invece narrazione aperta alla possibilità illimitata della poesia, dove il simbolo continuamente ri-velato dalle visioni di un bardo prescelto (come nel caso del "folle", "sciamanico" Tristano) permette la conoscenza di segreti riservati a pochissimi uomini.

Il discorso sul Graal, proprio per la presenza-assenza di un concetto/oggetto inafferrabile, è-per dirla con Foucault-non un "semplice intrecciarsi di parole e di cose" (Foucault 1980, 66), bensì una 
pratica (anche letteraria oltre che iniziatica), che "forma", sviluppa, l'oggetto di cui parla, attraverso "relazioni di significato" (Foucault I980, 67) che oltrepassano la lingua, il racconto, i topoi/luoghi, per appoggiarsi al sogno. La visione non è del resto meno veridica della realtà, il non-luogo in cui si colloca il Graal, ricercato ma irraggiungibile; sottratto ad ogni verifica razionale il Graal non è minore fonte di felicità (completa e perciò di per sé impossibile) rispetto alla conquista di un regno, di una donna e persino di Dio.

Ma il Medioevo delle forme (ricordiamo l'estetica morfologica di Henri Focillon: le forme $s i$ significano prima dei segni, e si generano/evolvono autonomamente) (Focillon 2OIO), il Medioevo della corporeità macerata dalla penitenza o sublimata dall'estasi, e nel contempo carnale, non poteva sottrarsi all'attrazione esercitata dalle reliquie della Passione (la croce, la lancia di Longino, il sudario), potenti media magico-simbolici del corpo vilipeso, assente eppure salvifico. È quella della "corporeizzazione del divino" (Zambon 2OI2, I42) un'esigenza non immune da influenze dogmatiche, prima fra tutte la necessità di affermare la reale presenza del Cristo nel sacrificio liturgico, solennemente dichiarata dal IV Concilio Lateranense (I2I5) che ispirerà nella Queste la concezione del Graal come sede della divina transustanziazione.

Frequente nei testi dell'epoca la corrispondenza tra gli oggetti liturgici e quelli della sepoltura di Cristo (potenza evocativa delle forme), come nel Joseph d'Arimathie o Roman de l'estoire dou Graal (ca. II9O-II99), romanzo in versi del poeta Robert de Boron, attivo tra XII e XIII secolo, l'altra celebre opera fondante il mito. La leggenda georgiana di Lydda, redatta tra il V e il VIII secolo ne fu una fonte ispiratrice e ci narra di Giuseppe d'Arimatea, recatosi sul Golgotha per raccogliere e custodire il sangue di Cristo imbevendone il lenzuolo funebre. Agli inizi del secolo XII la Gemma animae di Onorio Augustodunense, per influsso di un commentario liturgico bizantino anteriore di quattro secoli (la Historia ecclesiastica) aveva associato simbolicamente il calice della messa al sepolcro. Grazie alla successiva interpolazione dell'Historia, avvenuta tra XI e XII secolo, il calice diverrà la coppa in cui venne raccolto il sangue sgorgato dal costato trafitto del Crocifisso, e che ispirerà il Graal di de Boron (Zambon 2OI2, I5 ${ }^{\mathrm{I}-\mathrm{I}} 5^{2}$ ). Il Graal come purissima pietra ricomparirà nel Parzival di Wolfram von Eschenbach. Significativo quanto ha scritto recentemente il filologo francese Michel Zink, secondo il quale il Graal di de Boron non può essere identificato con il calice della messa, perché esso non fu sede di alcuna transustanziazione ma contenne il sangue reale dell'uomo Gesù (Zambon 2OI2, 236). Il rito del Graal istituito da Giuseppe d'Arimatea non era del resto una celebrazione eucaristica, ma la venerazione di una reliquia, di un Sacro Vaso, il più sacro di tutti i vasi.

Questa attenzione per i segni della Passione potrebbe non essere stata disgiunta da ragioni storiche contingenti. Elémire Zolla ha per esempio affermato con convinzione (e non senza azzardo) che il Re Pescatore, personaggio chiave del ciclo, sofferente per i peccati e sanabile solo dal cavaliere che ritroverà il Graal, simboleggia l'imperatore medievale d'Occidente, "impedito nella sua sacra funzione" e per questo impossibilitato a garantire pace sulla terra. La lancia, la coppa, la paténa ricordano gli oggetti liturgici della proskomidè, il prologo a porte chiuse della divina liturgia bizantina: un richiamo al bisogno dell'imperatore di riappropriarsi di una "regalità pontificale" costantiniana impedita dalla teocrazia cattolica. Dunque il Graal per Zolla sarebbe anche un archetipo politico. ${ }^{3}$

Associazioni simboliche, analogie, corrispondenze e ri-velazioni si accavallano, come se a rendere il "discorso sul Graal” fosse, quasi più delle interpolazioni testuali, il polimorfico quadro

3. Zolla I994, 98-99. Un’associazione, quella dell’immagine del Graal e della lancia alla liturgia ortodossa che è presente anche in Cristina Campo, quando descrive il pontificale bizantino per la vestizione del vescovo (Campo I97. I7O). 
culturale e dottrinale della civiltà medievale, cui si aggiunse una segreta tradizione apostolica, continuatrice del canone apocrifo scaturito dal Cristianesimo delle origini. Un passo nel prologo del Vangelo di Tommaso ci apre un nuovo piccolo spiraglio sulle parole "sacre e segrete" con le quali de Boron designa l'insegnamento di Gesù a Giuseppe d'Arimatea: "Colui che troverà l'interpretazione di queste parole non gusterà la morte" (Zambon 20I2, ,55). Che Gesù, prefigurando la rivelazione della sua gnosi, associ l'idea della morte al fatto che possa essere gustata, conferma come la prospettiva del sacrificio rimanga in ogni caso centrale, desiderata ed ineluttabile quantunque ritualizzata.

Dunque il Graal conduce non necessariamente alla salvezza, almeno in termini strettamente mondani, e ciò lo si evince dagli ultimi fatti della vita di re Artù. Al termine del Livre dou Graal (I2O5-I2IO Ca), trilogia in prosa attribuita a Robert de Boron, si narra del tradimento di Mordret, il nipote di Artù, al quale il re aveva affidato il regno durante la spedizione in terra francese. Mordret col suo gesto causerà la morte del sovrano senza che essa sia di riscatto per qualcuno (e il romanzo si era aperto col tradimento di Giuda). Come il sangue del Re dei re era stato portato nelle valli di Avalon, così il corpo ormai morente di Artù viene trasportato nell'omonima isola. Si realizza così, nel nome del Graal e attraverso la morte fisica, la compiuta comunione (teokoinia) tra Artù e Cristo. In una terra posta all'estremità dell'Occidente, il Graal ridiventa simbolicamente un sepolcro regale e non il suggello di una gloriosa incoronazione, sconfessando la profezia di Merlino, ritiratosi nei pressi del castello dove vive santamente Perceval. In questo scenario il Graal "sembra sprofondare di nuovo, insieme al suo re e al suo profeta, nelle tenebre di quel segreto da cui aveva avuto origine tutta la sua storia" (Zambon 2OI2, 200).

Questa oscurità col suo segreto ci sembra in qualche modo simile a quella generata dal Chaos primordiale che precede Bereshit, "la creazione da Dio stesso", cioè, come ha scritto Gershom Scholem $(1986,55)$, la creazione dal nulla, perché nulla potrebbe sussistere oltre a Dio all’inizio di tutte le cose, forse nemmeno il Graal. Il nulla e la pienezza dell'essere coesisterebbero così sin dall'Inizio per permettere al tutto di divenire, anche alla Verità.

Il Graal, indefinibile, onniassente "pietra di scandalo", è del resto simile alla verità al centro della celebre domanda rivolta da Pilato a Gesù nel Vangelo di Giovanni (I8,38): "Quid est veritas?”, "Che cos'è la verità?"”. Cristo non risponde e la verità resta un mistero aperto, desiderato e temuto, inconoscibile eppure reale come il contenuto del Graal. Pilato consegnerà Gesù al giudizio sommario della folla, lavandosi simbolicamente le mani: lavacro dell'ignavo "che mai non fu vivo", contrapposto al lavacro del cadavere dell'Innocente, morto per le colpe del mondo, il cui sangue verrà raccolto da Giuseppe d'Arimatea in un vaso, il Graal, lo stesso dell'Ultima cena, che, secondo la narrazione di de Boron, era stato consegnato da un ebreo a Pilato, il quale così ne divenne il momentaneo custode come lo fu del Cristo (Zambon 2OI2, 2O2).

E se la verità cercata da Pilato, un uomo chiamato a giudicare Dio, non fosse stata nient'altro che una delle "verità del Graal”? Cioè la Visio Dei? Quella che l'esoterista francese Pierre Ponsoye, allievo di Guénon, ha definito "non la visione di Dio da parte dell’uomo, ma la visione di Dio da parte di Dio stesso nell'uomo, il suo incontro con Se stesso nell'uomo, nel cuore dell'Istante eterno e del "divino Silenzio"” (Ponsoye r989, I3)? 
La sequenza metaformica del Graal, "Presenza vivente e permanente", ${ }^{4}$ pare non avere fine, ma ci sembra che il filo conduttore rimanga quello che conduce al sacrificio e, in ultima istanza, alla morte, alla quale non segue necessariamente un riscatto, una rinascita, come nel caso di Artù. Caso mai sono conseguenti e certe l'attesa e la speranza.

Se il Graal è "il referente ultimo verso il quale convergono tutte le interpretazioni allegoriche" (Zambon 20I2, 219), le avventure che attorno alla sua ricerca fioriscono, in una prospettiva misticoreligiosa, sono il complesso dei suoi segni, delle sue manifestazioni. Il senso letterale, non deve mai sottacere quello allegorico-simbolico e soprattutto "immaginale", ambiguo, quasi onirico. Sulla struttura narrativa del "romanzo graaliano" sono rimarchevoli le riflessioni del semiologo bulgaro Tzvetan Todorov (I97I, I29-I5O), per il quale non solo le aventures, ma anche le glosse, le intepretazioni rientrano nella cornice del racconto della Queste, e la ricerca del Graal diviene nient'altro che la "possibilità di un racconto" secondo una concatenazione: "Graal (Dio)-AvventuraRacconto". Una posizione non lontana da quella "razionalista” di Italo Calvino e Umberto Eco. Il critico francese Alexandre Leupin, ispirato a sua volta dal decostruzionismo di Derrida, ricorda come l'avventura del Graal sia votata al "vuoto" e alla "assenza del simulacro", due aspetti del racconto che "trovano il loro non-luogo nel Santo Vaso", "vaso eterodosso-scrive Leupin-che non si può riempire di senso, perché subito lo sottrae, contenitore incongruo che si sostituisce al calice per cancellare il Corpo sacramentale e farne un elemento supplementare della serie di fantasmi non totalizzabili; velo che svela solo il nulla [...]" (Zambon 2OI2, 222).

Insomma, sembra di comprendere che solo le "vie ingannevoli e fascinose" del racconto possono fornire una rappresentazione al Graal, solo la "potenzialità dell'ambiguo", che è propria dell'immaginario, e dunque della poesia come del romanzo, può renderci sopportabile il mistero che non riusciamo a svelare (Caillois I984, 99-IO5). Vorremmo però a questo punto osservare che il Graal può rendere possibile un discorso sulla funzione del romanzo che vada oltre le parole, oltre i significati, anche nella mitica prospettiva di una eudaimonia mai concretizzata e in sostanza antimetafisica.

Francesco Zambon, sottolineando la complessità simbolica della Queste, che "non ha molto da invidiare a quella della Commedia di Dante" (Zambon 20I2, 225), ritiene che gli strumenti critici adottati da Todorov e Leupin non siano però sufficienti. Per questo può essere d'aiuto il concetto di "racconto mistico" (récit mystique) nella definizione resa dal pensatore e orientalista francese Henry Corbin, la cui comprensione necessita di tre livelli: quello puramente teorico, quello in cui la "dottrina si fa evento", e quello della "realizzazione personale dell'evento" (Zambon 2or2, 226-227). Per tale ragione, un racconto come la Queste del Graal si comprende "nella misura in cui l'insegnamento teorico si è trasformato in evento e questo evento è stato "vissuto' personalmente". La "lettera del racconto" (le parole e le cose) non appartiene al mondo sensibile, visivo (quello del lettore), ma a quello "immaginale, "un mondo rigorosamente orientato", cioè del "ritorno all'Oriente", "centro" e "origine" del mondo e dell'essere, "il luogo in cui la conoscenza puramente dottrinaria diventa avvenimento" (Zambon 2OI2, 226-227).

Il senso del racconto sta perciò nella trasformazione individuale, nel completamento interiore, non immune dalla morte perché ogni antropo-poiesi è inserita nel tempo. Il Graal ci apparirà allora una sorta di "Evento" heideggeriano, l'Ereignis (Heidegger 2007): non un semplice accadimento,

4. Così scrisse nel I95 in un numero speciale dei Cahiers du Sud la medievista Myrrha Lot-Borodine (Ponsoye I989, I4). 
ma l'origine, cioè l'essere pensato nella sua verità all'interno del tempo che lo costituisce, tanto da "svanire" nell'Evento stesso ("Sein verschwindet im Ereignis") (Zarader I997, I68-I69). In questo perfezionamento/scomparsa nel tempo del nostro Esser-ci, il dono dell'immortalità che al kratêrgradalis-graal è attribuito già dalla ritualità protostorica, non consiste nell'eterna giovinezza, ma in una rinascita simbolica dopo che il tempo individuale dell'uomo, il nostro tempo, si esaurisce in continuazione nella divenienza della vita cosmica. La ferita del Re Pescatore è insanabile, ma la libertà creatrice non si interrompe, perché la libertà dell'Inizio è sia infinita e incontrollabile possibilità, sia -come vedremo- necessità.

\section{5}

Un raffinato ed extra-vagante letterato come il francese Roger Caillois ha scritto in Au coeur de fantastique:

Vi è un universo, quello delle confuse aspirazioni dell'anima, delle sue trame, delle sue delusioni, che un'irriducibile oscurità preserva dal pericolo d'essere assorbito. Né le parole, né le immagini possono circoscrivere esattamente quelle realtà interiori indefinite e mutabili che scoraggiano ogni descrizione e figurazione (Caillois I984, 99).

Ebbene, dal momento che l'oscura ambiguità dello spirito non può essere descritta da parole o immagini, una funzione speciale la svolge la musica. Se Chrétien de Troyes fu, per così dire, l'inventore del Graal nel Medioevo letterario, Richard Wagner fu "il creatore del suo mito moderno", individuando in questo mito "quasi il luogo geometrico delle sue più mature riflessioni estetico-religiose” (Zambon 2OI2, 263). Di queste riflessioni l'opera Parsifal rappresenta la sintesi musicale.

Grazie a Wagner, il tema del Graal uscì da una “eclisse” in cui si era occultato intorno alla metà del tredicesimo secolo, dopo un dis-velamento letterario durato poco più di cinquant'anni. ${ }^{5}$ L'interesse del compositore tedesco per questo "archetipo" prese avvio durante un suo soggiorno a Parigi (non a caso città di reliquie crociate, Nova Jerusalem) nell'inverno I84I-I842, grazie ad un amico filologo di origine ebraica, Samuel Lehrs (I8o6-I843), fratello del più noto classicista Karl, che viveva nella capitale francese (Foster 20IO, 277).

Il tema graalico fece la sua comparsa in Lohengrin e in Tristano e Isotta, ma culminò nella composizione del Parsifal, tra il I877 e il ı882, ultima opera del maestro che morì nel febbraio I883 a Venezia. La trama del Parsifal si ispira a Wolfram von Eschenbach, con l'integrazione di alcuni elementi desunti da altre opere del ciclo. Al centro del racconto, oltre al protagonista, stanno il Graal, identificato col calice in cui Giuseppe d'Arimatea raccolse il sangue sgorgato dal costato di Gesù crocifisso, e Amfortas, il re infermo, ferito nel petto dalla Sacra Lancia-quella di Longinodopo aver ceduto alla seduzione di Kundry, personaggio creato dal genio wagneriano, incarnante una potente ambiguità perché da un lato è la tentatrice del re, dall'altro suscita in Parsifal il dovere della missione redentrice. Wagner nel desiderio di conoscere la "profonda verità" del mito, e nella consapevolezza della caducità del mondo, dalla quale ci si deve affrancare superando la "volontà di vivere" che anima l'uomo istintuale, invoca una nuova coscienza. Essa può essere acquisita solo attraverso la conoscenza della compassione ("durch Mitleid wissend"), un concetto che rimanda al

5. Zambon $(2 \mathrm{OI} 2,263)$ sottolinea come l'anonimo autore della Queste, alla fine del romanzo fa rapire il Graal in cielo per volere divino in seguito alla morte del suo ultimo custode Galaad. 
profondo interesse di Wagner per gli insegnamenti del buddismo. Il redentore è colui che attraverso il dolore si identifica con tutte le creature sofferenti e inverte la "volontà di vivere", perché si sottrae al ciclo della "terribile causalità di ogni nascita e di ogni morte" (Zambon 2OI2, 264-267), superando il tempo e l'evento per costruire un mito rinnovato. Questo superamento fu reso possibile proprio dalla musica, che è oltre il tempo e lo spazio, prima di ogni intuizione, contro ogni illusione, perilmito.

Come osservò un fine interprete musicale quale Giuseppe Sinopoli in quella sorta di pamphlet iniziatico intitolato Parsifal a Venezia, definito da Cesare De Michelis "storia di uno smarrimento e soprattutto del lento, faticoso riconoscimento del senso e della direzione” (De Michelis 2002, IO), il tempo nel Parsifal è come sospeso e "allontana quel contaminante senso morale che vi è presente: diventa pura forma" (Sinopoli 2002, I5). Esso, come sembra rivelarci la sacra cerimonia iniziale del Graal, "altro non è che la rievocazione, attraverso il rito, dell'atto della Creazione", all'interno di uno spazio sacro che è un tutt'uno col tempo. Gurnemanz, il cavaliere che rivela come la redenzione verrà da un "casto folle" e che avvia Parsifal alla missione per il Graal, accompagnandolo nella sala del Graal esclama infatti: "Du siehst, mein Sohn, zum Raum wird hier die Zeit" (Vedi, figlio mio, il tempo qui diventa spazio). ${ }^{6}$ Claude Lévi-Strauss in questa frase ravvisò com’è noto la "definizione più profonda che sia mai stata data del mito" (Lévi-Strauss 2OIO, 223). Il celebre antropologo francese era del resto un estimatore dell'opera wagneriana, tanto che in un libro-intervista affermò: "Non soltanto Wagner ha costruito le sue opere sui miti, ma dei miti propone una scansione che l'uso del tema principale (il Leitmotiv) rende esplicita: il tema principale configura il mitema" (Eribon ed. 1988, 24I). Per l'autore di Tristes tropiques, il Leitmotiv wagneriano presentava probabilmente una funzione strutturalista, anche se il Graal, proprio per la sua natura archetipica, precede ogni "struttura", come ogni categoria interpretativa.

\section{6}

Lungo la scia delle visioni wagneriane, il Graal come "segreto che riguarda la morte" (Zambon 2OI2, 309) compare nella pubblicistica sul cosiddetto "Graal pirenaico", scaturita dalla identificazione della montagna sacra del Graal, Montsalvat, con Montségur, nei Pirenei, operata dallo scrittore ed esoterista francese Joséphin Péladan (I858-I9I8). Durante gli anni Trenta del "9oo, attraversati da tensioni mistico-escatologiche (preceduti e seguiti da conflitti mondiali), il mito del Graal fu rialimentato dal noto libro dello scrittore e "ariosofo" tedesco Otto Rahn (I904-I939), Crociata contro il Graal (Kreuzzug gegen den Gral) pubblicato a Friburgo nell'annus horribilis I933. Ad esso è legata l'eterodossa associazione del Sacro Vaso all'iniziazione catara, avente per teatro il sud della Francia, lo stesso che vide il martirio degli albigesi e la conquista di Montségur, ultima roccaforte dell'eresia, nel I244. I lavori di pubblicisti come il nazista Rahn, che morirà in circostanze misteriose dopo aver aderito alle $\mathrm{SS}$, si rivelano però estremamente fragili sul piano critico per le eccessive fantasticherie e le azzardate associazioni simboliche per nulla avvalorate sul piano storico e filologico (Zambon, 20I2, 2I5 ss.).

Doveroso piuttosto ricordare che il revival del Graal (e del catarismo) affascinò-con ben altri propositi ed esiti-anche una straordinaria pensatrice come Simone Weil nel corso della sua intima

6. Kurt Hübner ha scritto che Wagner riesce ad inserire anche il mondo privo di ogni trascendenza o caratterizzato dai dogmatismi religiosi in una realtà puramente mistica, sottratta alle coordinate del presente. Egli in questo modo "mostra al tempo stesso la radice numinosa di tale presente storico", e "allude ad un accadere che è tanto al di sopra del tempo quanto alla base di esso” (Hübner I99O, 447). 
ricerca-attesa di Dio, ispirandole una serie di pregnanti riflessioni sul concetto di assimilazione dell'uomo a Dio attraverso il dolore (Gianfreda 2OI2). Riferendosi alle figure del Re Pescatore e di Parsifal, scrisse la Weil:

La pienezza dell'amore del prossimo sta semplicemente nell'essere capace di domandargli: “Qual è il tuo tormento?", nel sapere che lo sventurato esiste, non come uno fra i tanti [...] ma in quanto uomo, in tutto simile a noi, che un giorno fu colpito e segnato dalla sventura con un marchio inconfondibile. Per questo è sufficiente, ma anche indispensabile, saper posare su di lui un certo sguardo (Weil 1984, 83).

Quello evocato dalla Weil non è uno sguardo qualsiasi, ma “attento", nel quale "l'anima si svuota di ogni contenuto" (quasi-diremmo noi-in una sorta di kenosi, di rinunzia del sé per l'altro, di atto creativo) “per accogliere in sé l'essere che essa vede così com'è nel suo aspetto vero" (Weil I984, 83-84). Ė forse questo sguardo simile a quello di Gesù, che chiama e perdona, nei Vangeli? In realtà è il frutto di un'attenzione e di uno sforzo ancor maggiori, perché esclusivamente terreni, anche se l'amore di Dio e degli uomini "è fatto della stessa sostanza”. Simone Weil scrive infatti che la capacità di prestare attenzione è una capacità "difficilissima", "rarissima": letteralmente un "miracolo" (Weil I984, 83) (come l'azione del Graal), una scelta che si oppone all'uccisione delle anime provocata da coloro che "fanno precipitare nella sventura esseri umani non preparati a sopportarla" (Weil I984, 88).

La disperazione delle anime è l'esito di un atteggiamento anti-creativo, la conseguenza del male che l'uomo "porta in sé", distruggibile solo dalla "presenza sensibile della perfetta purezza". Questa purezza è quanto distingue i nuovi katharoi che orientano la loro anima verso Dio nonostante la sofferenza, mentre un "un chiodo" li trafigge "al centro dell'universo", il "vero centro", "che è fuori dello spazio e del tempo, che è Dio" (Weil I984, IOI). Questo centro, al quale Dio "s'incatena mediante la necessità" (Weil I995, 95) che gli deriva dalla libertà creatrice, è forse il luogo dove si trova il Graal. Simone Weil, pur occupandosene nell'ultima fase della sua esistenza, ${ }^{7}$ non aderì però al catarismo, perché nei suoi scritti la sofferenza divina è comunque redentrice, e "anche la creazione è passione" (Zambon r997, 24-25). Infatti la sofferenza non era per Simone Weil una necessità dell'uomo allo scopo di separare l'anima dal corpo in ossequio alla visione dell'irrimediabile corruzione della materia creata, ma una comune condizione dell'uomo e di Dio "per effetto della necessità". Anzi, la pensatrice arrivò a scrivere che la "materia che ha causato la perdizione procura la salvezza", della quale il corpo è "potente strumento" tanto che si "desidera essere semplicemente una parte del mondo" (Weil I993, 263), piuttosto che se stessi. Una posizione questa decisamente lontana dalla teologia catara e dalla visione dualistica in generale. Il messaggio di Simone Weil fu perciò un atto di fede e di pietas radicale, frutto di quel suo sguardo così intenso, attento sull'uomo la cui portata universale non avvertì il bisogno del battesimo per perfezionarsi.

\section{7}

L'epos cavalleresco del Graal ricompare nel Novecento letterario italiano in poeti e scrittori come Arturo Onofri, Italo Calvino o Umberto Eco. Sulla scorta di alcune significative pagine del volume di Zambon (Zambon 20I2, 27 I-283; 345-372), vogliamo soffermarci in particolare su uno scrittore razionale e fantastico come Calvino, nel quale l'uso frequente dell'allegoria e del simbolo, assieme all'adozione di un impianto narrativo fiabesco, si propone di suscitare il contrasto tra

7. Si vedano i suoi saggi (Weil I996), e apparsi nei Cahiers du Sud nel I942. 
realtà e illusione, tra la ragione e il mito, ${ }^{8}$ oltre ogni verità assoluta e lontano dai giochi ermeneutici del "cerebrale" Eco. Ne è un esempio un episodio de Il cavaliere inesistente, racconto del 1959. L'impianto fiabesco e l'ambientazione in un medioevo ambiguo quanto irreale ci offrono la prima vera e sottile "caricatura" del Graal. Calvino pare smitizzare il mito, superando, anzi sconfessando la sua "valenza redentrice" come la sua appartenenza alla "tradizione primordiale" cara ad autori come Guenon ed Evola. L'ordine dei cavalieri del "Gral” (sic) somiglia ad una "sgangherata setta orientaleggiante dei nostri giorni" (Zambon 2OI2, 346), mossa dalla cosiddetta "forza del Gral”, lacerata tra una perdita dell’individualità per fondersi col Cosmo e la volontà di operare determinate scelte, anche immorali. Ne Il castello dei destini incrociati, del I973, il Graal diventa il "centro vuoto della ricerca senza fine di un centro e di una verità", "il nucleo cavo del mondo", "l'assenza intorno alla quale tutto si costruisce" (Zambon 2OI2, 37I), insomma un segreto vuoto "riempibile di qualunque senso", come sentenzierà quindici anni più tardi Umberto Eco ne Il pendolo di Foucault (I988) (Zambon 20I2, 36I). Ad una recensione di Walter Pedullà che scorgeva nell'ordine dei cavalieri del "Gral" una parodia dei comunisti, Calvino rispose ${ }^{9}$ rinviando ad un suo saggio, Il mare dell'oggettività, apparso su Menabò nel febbraio del r96o. In una lettera aperta lo scrittore affermò di essere stato ispirato dalla "tendenza verso l'oggettività" che segnava in quegli anni la cultura e la società, così dissimile dal "flusso della soggettività" joyceana, espressionista e surrealista che nel primo Novecento aveva segnato anche la letteratura italiana (Zambon 20I2, 346-347). Due intellettuali italiani incarnavano bene la nuova "tendenza verso l'oggettività": Pietro Citati ed Elémire Zolla.

Questa "tendenza" simile ad "una completa comunione col tutto", dove l’Io si annega nella storia o nella natura, è per Calvino analoga alla mistica praticata dai cavalieri del "Gral”, e somiglia all'aristocratico isolamento praticato da certi astratti intellettuali, così lontano dal suo ideale eticoartistico fatto di romanzi con al centro le azioni di personalità morali, simili a quei personaggi delle fiabe che superano difficilissime prove (Zambon 2OI2, 348-349).

Agli occhi di Calvino, la "cerca" del Graal appare la continuazione di una ricerca di senso e di "verità" ostinata quanto votata al fallimento. Se da un lato il suo razionalismo mette in discussione gli ideali etico-religiosi di Wagner e dell'irrazionalismo europeo in genere (di cui il Graal è millenario quanto rischioso paradigma letterario), dall'altro egli ritiene che "la Verità ultima dell'universo" sia una combinazione infinita di elementi attorno ad un "centro che è puro vuoto, puro nulla”. Quest’ultima del resto è per Calvino la verità della letteratura, e personaggi del Castello come Faust o Parsifal "sono già incarnazioni dello scrittore" (Zambon 2012, 354). ${ }^{\circ}$

Se la visione smitizzante di Calvino non impedisce comunque di attribuire alla ricerca narrativa del Graal una valenza gnoseologica per comprendere la condizione umana (in Calvino la letteratura ha una "funzione esistenziale") (Lollini I997, 284), in Eco lo "svuotamento radicale del mito del Graal” appare maggiormente l'espressione di un "gioco ermeneutico", nel quale si rifiuta come irrazionale tutto ciò che esula dai modelli semiologici dei suoi scritti, pressoché ignorando ("o forse ostentando di ignorare") le fonti letterarie medievali e indugiando su certa vulgata occultistica (Zambon 2OI2, 36I-366).

8. Secondo Massimo Lollini, “per Calvino la letteratura scritta è radicata nel mito e nel rito, e mantiene con queste lontane realtà un rapporto di continuità sul terreno antropologico. Calvino è convinto che nel mondo del mito la letteratura è nata con un compito di consacrazione dell'esistente, senza svolgere alcuna funzione critica; e che ci sono voluti millenni per liberare la letteratura da questo compito conservatore e tradizionale" (Lollini, I997, I5, 284).

9. Su Mondo Nuovo, 3 aprile ig6o.

Io. Al termine de Il cavaliere inesistente, Suor Teodora alias Bradamante afferma: "La pagina ha il suo bene solo quando la volti e c'è la vita dietro che spinge e scompiglia tutti i fogli del libro”(Calvino 2OII, 4O7). 
Il Graal è forse ormai relegato ad una prospettiva non più trascendente o mitologica, costretto all'interno di recinti mimetici, puramente teorici e narrativi dove la "pienezza dell'essere" rimane un’utopia che si trasforma "in un'ulteriore riflessione sul nulla" (Lollini 1997, 303). Se ciò fosse, è per questa ragione che il millenario cammino del Graal, divenuto un "fantasma [...] che innesca i meccanismi del desiderio e del racconto" (Lollini 1997, 372), è forse giunto alla sua fine?

Vogliamo credere che il percorso del Graal non sia terminato, e questa affermazione non muove da una disperata sfida irrazionale ai postulati della gnoseologia, ma nasce dalla consapevolezza di quanto sia importante nella conoscenza del mundus imaginalis, che è della mistica come della letteratura, quella che Corbin chiama la "percezione immaginativa”. Nel profetismo islamico, oggetto della percezione, anche di un oggetto sensibile, tangibile, sono "le forme che l'anima vede con l'occhio della coscienza immaginativa" (Corbin I973,69). Non è questo esclusivamente l'esito di una illuminazione iniziatica, ma una visione che non pare così lontana da quella concezione filosofica occidentale e propriamente "razionale" che dai sofisti arriva ad Husserl.

Certamente, se è difficile la conoscenza di un "oggetto" in perenne metamorfosi come il Graal, meno ardua è la sua sperimentazione in quel "terzo mondo" (immaginale) "intermediario tra il mondo sensibile e il mondo del puro intelligibile”, attraverso il quale-osserva Corbin-è possibile sfuggire alle maglie sia dell'astratto spiritualismo dei filosofi sia del "letteralismo dei teologi" (ricordiamo il dogmatismo eucaristico), per approdare ad una "verità spirituale letterale" (Corbin I973, I89). Corbin nei suo scritti si nutriva della tradizione mistica e filosofica persiana oltre che rifarsi alla gnosi di matrice islamica, ma ciò non impedisce di ricercare anche nel pensiero dell'Occidente una possibilità o meglio un ulteriore senso per tale "verità".

Per questo vorrei concludere il mio intervento con il "pensiero poetante" di Heidegger, un filosofo che col mito e la gnosi ha avuto un indubbio legame (Moretto 2OO2, II73-II92). Per il "mago di Messkirch”, come per Platone, mythos e lógos sono strettamente correlati (Moretto 2002, II9O), e la dimensione della Geworfenheit (l'essere-gettato, condizione della nostra esistenza in un mondo che viene prima di noi e con cui dobbiamo rapportarci) somiglia alla dottrina gnostica mandea dell'essere-gettato intesa come separazione della creatura dal principio creatore (Moretto 2002, II78).

Nella lirica Gefährten (Compagni di strada), compresa nella raccolta Winke (Cenni) del I94I, forse possiamo cogliere qualche indicazione per immaginare un cammino ( $W e g$ ) alternativo (che è forse lo stesso da sempre) per il Graal.

Scrive Heidegger che: "essere è evento / evento è inizio / inizio è portare a conclusione / portare a conclusione è congedo / congedo è essere" "Seyn ist Ereignis / Ereignis ist Anfang / Anfang ist Austrag / Austrag ist Abschied / Abschied ist Seyn). La storia del mondo ci appare in questa lirica come la sequenza di un continuum temporale e creativo: ogni Creazione è fatta di ininterrotti nuovi inizi come di nuove, continue conclusioni, di addii (congedi) che diventano ripresenze nel mondo, perché ogni evento, l'essenza dell'essere, è “la storia più originaria” (Cassinari 2000, IOO-IO2), il racconto dell'Inizio.

Il mito nella sua lontana "divenienza" ed "evenienza" appare perciò una rappresentazione simbolica del processo creativo, che va oltre ogni racconto, oltre il nostro esserci, che si congeda persino dalla storia senza che l'essere venga messo in dicussione. Il Graal, nella sua mutevole,

II. Cassinari 2000, 72-73. Flavio Cassinari traduce Austrag con 'conclusione che diverge'. Il termine Austrag è tradotto invece da Gianni Vattimo (Heidegger I976, I5I) con ‘diaferenza'. 
millenaria continuità, appare perciò un insostituibile "compagno di strada", senza il quale all'uomo risulterebbe più difficilmente praticabile quella temuta "intimità col divino" che per $\mathrm{i}$ "sensi umani"-osservò l'indimenticata Cristina Campo-costituisce una "suprema occasione": "l'occasione della metamorfosi" (Campo 1987, 231).

\section{Opere citate}

Cacciari, Massimo, 2004, Della cosa ultima, Milano, Adelphi.

Caillois, Roger, 1984, Nel cuore del fantastico, Milano, Feltrinelli (Au coeur du fantastique, 1965).

Calvino, Italo, 2OII, Il cavaliere inesistente, nel suo Inostri antenati, Milano, Mondadori.

Campo, Cristina, 1971, Il flauto e il tappeto, Milano, Rusconi.

Campo, Cristina, 1987, Gli imperdonabili, Milano, Adelphi.

Cassinari, Flavio, 2000, Martin Heidegger, il pensiero poetante: la produzione lirica heideggeriana (1910-1975), Milano, Mimesis.

Corbin, Henry, I973, Storia della filosofia islamica, Milano, Adelphi (Histoire de la philosophie islamique, 1964).

De Michelis, Cesare, 2002, "Per Giuseppe Sinopoli”, in Giuseppe Sinopoli, Parsifal a Venezia, Venezia, Marsilio.

Dumézil, Georges, I968, Mythe et épopée. L'idéologie des trois fonctions dans les épopées des peuples indo-européens. Paris, Gallimard.

Focillon, Henri, 20IO, Vie des formes, Paris, PUF.

Foster, Daniel H., 20IO, Wagner's Ring Cycle and the Greeks, Cambridge, Cambridge University Press.

Foucault, Michel, I980, Archeologia del sapere, Milano, Rizzoli (L'archéologie du savoir, I969).

Gianfreda, Fausto, 20I2, Il Graal di Simone Weil, Verucchio (RN), Pazzini.

Givone, Sergio, 2006, Storia del nulla, Roma-Bari, Laterza.

Heidegger, Martin, 1976, Saggi e discorsi, trad. Gianni Vattimo, Milano, Mursia.

Heidegger, Martin, 2007, Contributi alla filosofia (Dall'evento), Franco Volpi ed., Milano, Adelphi (Beiträge zur Philosophie (Vom Ereignis), 2003).

Hübner, Kurt, 1990, La verità del mito, Milano, Feltrinelli (Die Wahrheit des Mythos, 1985).

Jesi, Furio, r989, Mito, Milano, Mondadori.

Lévi-Strauss, Claude, 20Io, Lo sguardo da lontano, Milano, Il Saggiatore (Le regard éloigné, I983).

Lévi-Strauss, Claude, I988, Da vicino e da lontano, Didier Eribon ed., Milano, Rizzoli (De près et de loin. Entretiens avec Claude Lévi- Strauss, 1988).

Lollini, Massimo, 1997, "Antropologia ed etica della scrittura in Italo Calvino", Annali d'Italianistica 
I5, 283-3II. http://tinyurl.com/npxmzys

Moretto, Giovanni, 2002, Il mito in Heidegger tra esistenza ed evento, in La persona e i nomi dell'essere: scritti di filosofia in onore di Virginio Melchiorre, Francesco Botturi, Francesco Totaro, Carmelo Vigna, ed., Milano, Vita e Pensiero, vol. 2, II77-II92.

Ponsoye, Pierre, I989, L'Islam e il Graal, Milano, SE (L'Islam et le Graal, I957).

Scholem, Gershom, I986, Concettifondamentali dell'ebraismo, Genova, Marietti (Die jüdische Mystik in ihren Hauptströmungen, 1957).

Sinopoli, Giuseppe, 2002, Parsifal a Venezia, Venezia, Marsilio.

Todorov, Tzvetan, 1971, Poétique de la prose, Paris, Seuil.

Walzer, Michael, 1985, Exodus and revolution, New York, Basic Books.

Weil, Simone, 1984, Attesa di Dio, Joseph-Marie Perrin ed., Milano, Rusconi (Attente de Dieu, I950).

Weil, Simone, I985, Quaderni II, Giancarlo Gaeta ed., Milano, Adelphi (Cahiers II, I953).

Weil, Simone, 1996, I catari e la civiltà mediterranea, Genova-Milano, Marietti.

Weston, Jessie L., I994, Indagine sul Santo Graal: dal rito al romanzo, Pallermo, Sellerio (From ritual to romance, $\mathrm{I} 92 \mathrm{O})$.

Zambon, Francesco, 1997, "Introduzione”, in La cena segreta: trattati e rituali catari, Francesco Zambon ed., Milano, Adelphi.

Zambon, Francesco, 2012, Metamorfosi del Graal, Roma, Carocci.

Zarader, Marlène, I997, Heidegger e le parole dell'origine, Milano, Vita e Pensiero (Heidegger et les paroles de l'origine, 1990)

Zolla, Elémire, I994, Archetipi, Venezia, Marsilio (Archetypes, 198I). 